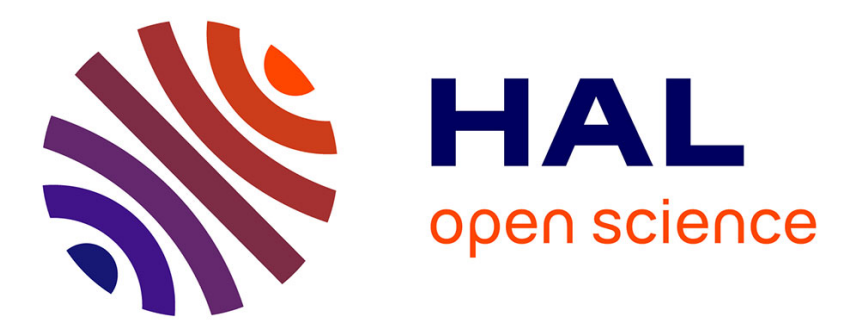

\title{
Comparaison des rendements de croissance sur lactose de quelques Kluyveromyces Van der Walt
}

\author{
J. Ladet, G. Moulin, P. Galzy, J. L. Joux, F. Biju-Duval
}

\section{To cite this version:}

J. Ladet, G. Moulin, P. Galzy, J. L. Joux, F. Biju-Duval. Comparaison des rendements de croissance sur lactose de quelques Kluyveromyces Van der Walt. Le Lait, 1972, 52 (519_520), pp.613-621. hal-00928604

\section{HAL Id: hal-00928604 https://hal.science/hal-00928604}

Submitted on 1 Jan 1972

HAL is a multi-disciplinary open access archive for the deposit and dissemination of scientific research documents, whether they are published or not. The documents may come from teaching and research institutions in France or abroad, or from public or private research centers.
L'archive ouverte pluridisciplinaire HAL, est destinée au dépôt et à la diffusion de documents scientifiques de niveau recherche, publiés ou non, émanant des établissements d'enseignement et de recherche français ou étrangers, des laboratoires publics ou privés. 


\title{
Comparaison des rendements de croissance sur lactose de quelques Kluyveromyces Van der Walt
}

\author{
par \\ J. LADET, G. MOULIN, P. GALZY, J. L. JOUX* \\ et F. BIJU-DUVAL* \\ Laboratoire de Recherches de la Chaire de Génétique \\ Ecole Nationale Supérieure Agronomique \\ Centre de Recherches Agronomiques de Montpellier \\ Institut National de la Recherche Agronomique \\ (34) Montpellier (France) \\ et \\ *Centre de Recherches des Fromageries Bel \\ (41) Vendôme (France)
}

De nombreuses espèces de Kluyveromyces fermentent le lactose. Parmi celles-ci, Kluyveromyces fragilis (Jorgensen) Van der Walt est utilisée en laiterie. Il est en effet possible de cultiver cette levure sur lactosérum et d'obtenir ainsi une levure-aliment de bonne qualité (1).

Ce travail a pour but de comparer les rendements de croissance sur lactose de diverses souches de Kluyveromyces.

Rappelons que les Kluyveromyces étaient classés jusqu'à une date récente parmi les Saccharomyces. Ce genre a été divisé en deux groupes distincts à la suite des travaux de Van der Walt (1970).

\section{I. - MATERIEL ET METHODES}

\section{a) Matériel biologique}

Six souches ont été utilisées. Elles proviennent de diverses collections :

CBS : Centraalbureau voor Schimmelcultures, Delt.

STV : Station de Technologie Végétale, Dijon.

SO : Station Oenologique, Bordeaux.

SG : Service de Génétique, E.N.S.A., Montpellier.

(1) Un brevet a été pris (brevet $\mathrm{n}^{\circ} 1128063$ des fromageries Bel). 
Ces souches sont les suivantes :

K. marxianus (Hansen) Van der Walt : STV 1043

$K$. lactis (Dombrowski) Van der Walt : SO

$K$ fragilis (Jorgensen) Van der Walt : $\quad$ SG 1

$K$ fragilis (Jorgensen) Van der Walt : $\quad$ CBS 397

$K$ fragilis (Jorgensen) Van der Walt : CBS 1555

$K$ fragilis (Jorgensen) Van der Walt : $\quad$ CBS 5795

\section{b) Techniques de culture}

Toutes les cultures sont effectuées sur milieu yeast nitrogen base Difco avec du lactose comme source de carbone. Elles sont agitées à $25^{\circ} \mathrm{C}$ ( 80 oscillations $/ \mathrm{mn}$; amplitude $\left.8 \mathrm{~cm}\right)$ dans des erlenmeyers.

\section{c) Techniques analytiques} (1951).

Les protéines sont dosées par la réaction du biuret selon Strickland

La matière sèche est mesurée par pesée après dessiccation à $108^{\circ} \mathrm{C}$ jusqu'à poids constant.

Les lipides totaux, l'insaponifiable et le saponifiable sont extraits et dosés par les méthodes déjà décrites (A. Chassang et al. 1972).

Le nombre de cellules est estimé par comptage hématimétrique.

\section{II. - RESULTATS}

\section{a) Etude du rendement de croissance sur lactose}

Le rendement de croissance a été étudié en faisant varier la teneur en lactose de 0,1 p. 100 à 2 p. 100 . La culture est conduite en erlenmeyers de $100 \mathrm{ml}$ remplis au $10^{\mathrm{e}} \mathrm{du}$ volume. En fin de croissance, un comptage hématimétrique et un dosage des protéines sont effectués.

Le graphique $\mathrm{n}^{\circ} 1$ donne un exemple des résultats obtenus : Souche $K$. fragilis SG 1 . Le seul facteur limitant la biosynthèse des protéines est le lactose jusqu'à la concentration de 0,8 p. 100 .

Les concentrations supérieures ne donnent plus d'augmentation de la biosynthèse des protéines car d'autres facteurs limitants interviennent. Si la croissance est exprimée par le nombre de cellules, on constate que la multiplication cellulaire n'est proportionnelle à la concentration en lactose que jusqu'aux environs de 0,5 p. 100. Le nombre de cellules et la quantité de protéines augmentent simultanément en fonction de la concentration en lactose pour les teneurs inférieures à 0,5 p. 100 . Entre 0,5 et 0,8 p. 100 , la biosynthèse des protéines continue à être proportionnelle à la teneur en lactose, alors que la multiplication cellulaire est arrêtée. Cette anomalie se retrouve dans toutes ces expériences et pour toutes les souches. 
Les cellules des Kluyveromyces semblent avoir des tailles variables en fonction des conditions de culture. La richesse en lactose du milieu, et, peut être aussi les équilibres alimentaires, conditionnent les mécanismes régulateurs de la multiplication cellulaire et de la biosynthèse des protéines.

Nous avons représenté dans le graphique $\mathrm{n}^{\circ} 2$ le résultat obtenu avec $K$. marxianus STV 1043. En effet, cette souche présente une légère différence de comportement par rapport aux autres. Au-dessus de 0,8 p. 100 de lactose, la biosynthèse des protéines continue à augmenter très légèrement avec la richesse en lactose. Cette augmentation est faible et s'effectue avec un très mauvais rendement. Elle traduit peut-être une particularité du métabolisme de $K$. marxianus.

Ce type d'expérience a été effectué plusieurs fois pour chaque souche. Les résultats sont résumés dans le tableau $n^{\circ} 1$. Nous donnons d'abord les rendements en $\mathrm{mg}$ de protéines et en nombre de cellules par mg de lactose consommé lorsque le lactose est le seul facteur limitant la croissance ( $1^{\text {re }}$ et $2^{\text {me }}$ colonne). Ces rendements sont déterminés en calculant la pente des droites de régression pour la zone de concentrations inférieures à 0,8 p. 100 ou 0,5 p. 100. Nous donnons

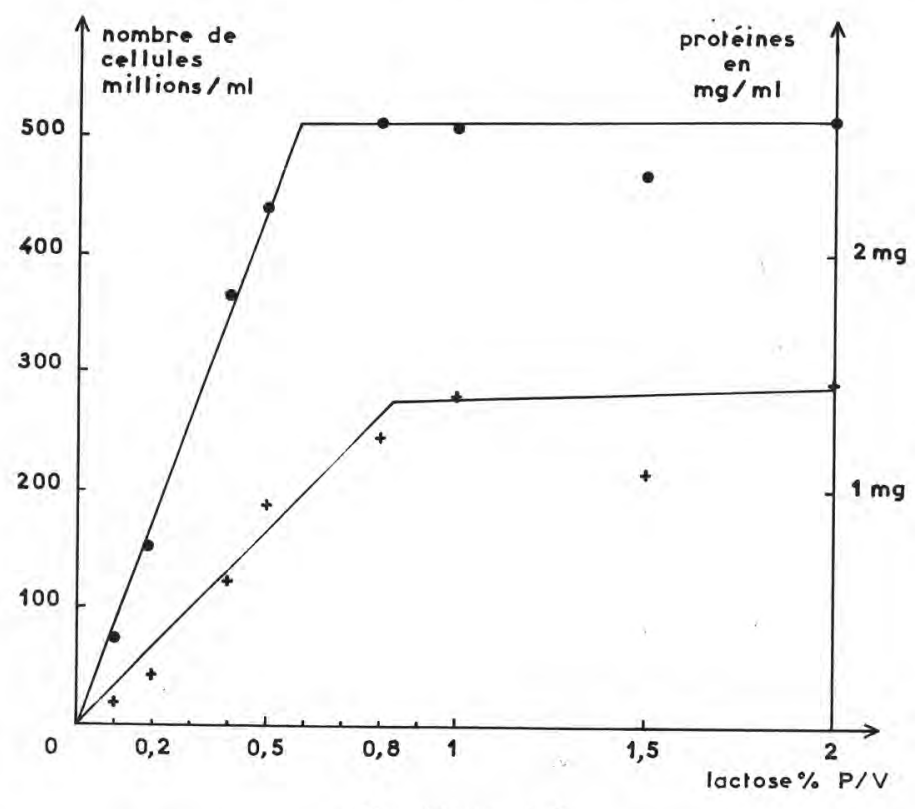

Graphique $n^{\circ} 1$

Rendement de croissance en présence de quantités variables de lactose Souche Kluyveromyces fragilis SG 1

- Nombre de cellules par ml de culture exprimé en millions.

+ Protéines par $\mathrm{ml}$ de culture exprimées en mg. 


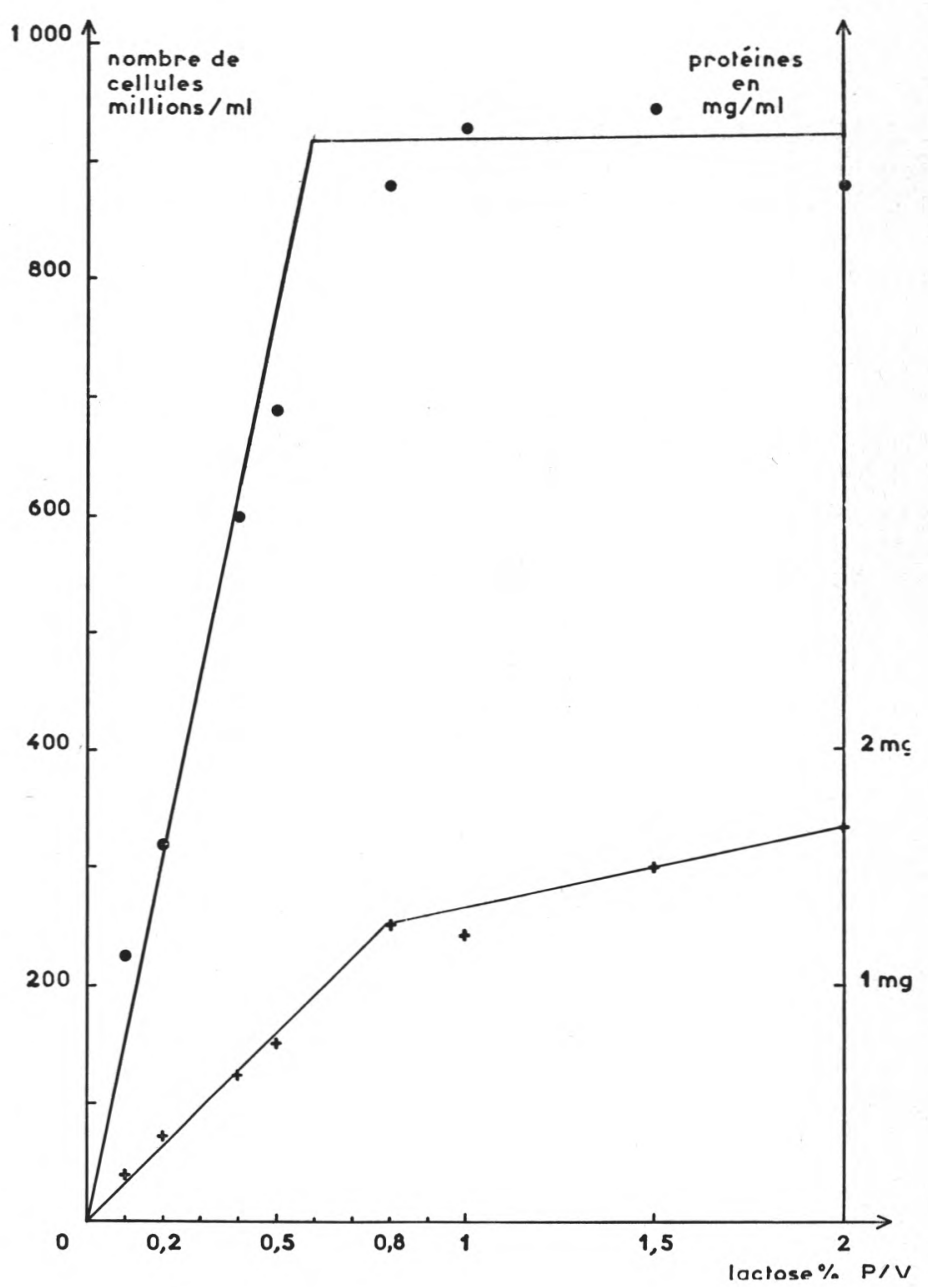

Graphique $n^{\circ} 2$

Rendement de croissance en présence de quantités variables de lactose Souche Kluyveromyces marxianus STV 1043

- Nombre de cellules par ml de culture exprimé en millions.

+ Protéines par ml de culture exprimées en mg. 


\section{TABLEAU 1}

\begin{tabular}{|c|c|c|c|c|c|c|}
\hline \multirow{2}{*}{ Souches } & \multicolumn{2}{|c|}{ Rendement par mg de lactose } & \multicolumn{2}{|c|}{$\begin{array}{l}\text { Croissance maximum sur le milieu } \\
\text { Yeast Nitrogen Base Lactose }\end{array}$} & \multicolumn{2}{|c|}{ mg de protéines dans $10^{8}$ cellules } \\
\hline & $\begin{array}{l}\mathrm{mg} \mathrm{de} \\
\text { protéines }\end{array}$ & $\underset{\left(\times 10^{8}\right)}{\text { Cellules }}$ & $\begin{array}{l}\text { Nombre de } \\
\text { cellules }\left(\times 10^{s}\right)\end{array}$ & $\begin{array}{l}\text { mg de } \\
\text { protéines }\end{array}$ & $\begin{array}{l}\text { Lactose limitant } \\
\text { la croissance }\end{array}$ & $\begin{array}{l}\text { Lactose en } \\
\text { excès }\end{array}$ \\
\hline $\begin{array}{l}\text { Kluyveromyces } \\
\text { fragilis SG } 1\end{array}$ & 0,17 & 0,8 & 4,2 & 1,4 & 0,21 & 0,33 \\
\hline $\begin{array}{l}\text { Kluyveromyces } \\
\text { fragilis } 5795\end{array}$ & 0,16 & 0,6 & 3,7 & 1,3 & 0,26 & 0,35 \\
\hline $\begin{array}{l}\text { Kluyveromyces } \\
\text { fragilis } 1555\end{array}$ & 0,13 & 0,65 & 3,2 & 1,3 & 0,20 & 0,41 \\
\hline $\begin{array}{l}\text { Kluyveromyces } \\
\text { fragilis } 397\end{array}$ & 0,13 & 0,5 & 3,0 & 1,1 & 0,26 & 0,37 \\
\hline $\begin{array}{l}\text { Kluyveromyces } \\
\text { lactis }\end{array}$ & 0,10 & 0,7 & 3,2 & 0,7 & 0,14 & 0,22 \\
\hline $\begin{array}{l}\text { Kluyveromyces } \\
\text { marxianus }\end{array}$ & 0,11 & 1,5 & 10 & 1,5 & 0,11 & 0,15 \\
\hline
\end{tabular}


ensuite la croissance maximum obtenue sur le milieu yeast nitrogen base exprimée en nombre de cellules et en $\mathrm{mg}$ de protéines lorsque le lactose n'est plus limitant. Les valeurs caractérisent les facteurs limitant la croissance dans le milieu autres que le lactose.

Enfin les deux dernières colonnes indiquent la richesse des cellules en protéines lorsqu'elles ont été obtenues en présence d'une quantité limitante de lactose d'une part, et d'un excès de lactose d'autre part.

Lorsque le lactose est le seul facteur limitant la croissance, le rendement en protéines varie de 0,10 à 0,17 . Compte tenu de la fluctuation biologique et des erreurs d'expériences, il est permis de conclure que les $K$. fragilis donnent un rendement supérieur aux autres Kluyveromyces. Bien que la souche $K$. fragilis SG 1 donne le meilleur rendement $(0,17)$, les différences entre les divers $K$. fragilis ne semblent pas significatives.

Le tableau no 1 met en évidence la grande variabilité de la taille des cellules selon la souche. $K$. marxianus a des cellules particulièrement petites. Les deux dernières colonnes du tableau $\mathrm{n}^{\circ} 1$ confirment les différences de taille des cellules entre les diverses souches. Elles montrent surtout que, dans ces expériences, pour toutes les souches, les cellules sont plus riches en protéines lorsque le lactose est en excès dans le milieu que lorsqu'il constitue le facteur limitant la croissance. Les cellules sont également de plus grande taille.

\section{b) Etude de la composition des cellules}

Il apparaît d'après les résultats ci-dessus que les conditions de milieux peuvent modifier la taille et la composition des cellules des Kluyveromyces. Nous avons donc étudié la composition des cellules dans deux conditions : lactose limitant la croissance : 0,2 p. 100, et lactose en excès : 0,8 p. 100. Dans les deux cas, nous avons utilisé des erlenmeyers de 61 contenant 11 de milieu. Les cultures sont agitées.

Le tableau $n^{\circ} 2$ indique les résultats obtenus ramenés à la matière sèche pour protéine, nombre de cellules et lipides totaux. Les teneurs en saponifiable et insaponifiable sont exprimées en pourcentage des lipides totaux.

Après culture sur lactose 0,2 p. 100, Kluyveromyces lactis SO présente une richesse en protéines supérieure aux autres souches. Par contre Kluyveromyces marxianus STV 1043 est particulièrement pauvre. Les 4 souches de Kluyveromyces fragilis présentent des richesses en protéines très voisines. La souche utilisée actuellement par les Fromageries Bel (5795) est la plus riche en lipides totaux et en acides gras. La souche Kluyveromyces fragilis SG 1 est au contraire la plus pauvre en lipides totaux et en acides gras ; elle contient, exprimée en pourcentage de la matière sèche, 2,4 p. 100 d'acide gras au 
TABLEAU 2

\begin{tabular}{|c|c|c|c|c|c|}
\hline Souches & $\begin{array}{l}\text { Protéines } \\
\text { en p. } 100 \text { de } \\
\text { matière } \\
\text { sèche }\end{array}$ & $\begin{array}{l}\text { Nombre de cellules } \\
\text { par mg de } \\
\text { matière sèche } \\
\left(\times 10^{8}\right)\end{array}$ & $\begin{array}{l}\text { Lipides totaux } \\
\text { en p. } 100 \text { de } \\
\text { matière } \\
\text { sèche }\end{array}$ & $\begin{array}{l}\text { Insaponifiable } \\
\text { en p. } 100 \text { des } \\
\text { lipides } \\
\text { totaux }\end{array}$ & $\begin{array}{l}\text { Saponifiable } \\
\text { en p. } 100 \text { des } \\
\text { lipides } \\
\text { totaux }\end{array}$ \\
\hline & \multicolumn{5}{|c|}{0,2 p. 100 de lactose } \\
\hline $\begin{array}{l}\text { Kluyveromyces } \\
\text { fragilis SG } 1\end{array}$ & 36 & 1,7 & 4,9 & 32 & 50 \\
\hline $\begin{array}{l}\text { Kluyveromyces } \\
\text { fragilis } 5795\end{array}$ & 39 & 1,4 & 7,3 & 19 & 57 \\
\hline $\begin{array}{l}\text { Kluyveromyces } \\
\text { fragilis } 1555\end{array}$ & 38 & 2,2 & 5,3 & 25 & 55 \\
\hline $\begin{array}{l}\text { Kluyveromyces } \\
\text { fragilis } 397\end{array}$ & 34 & 1,4 & 6,1 & 19 & 61 \\
\hline $\begin{array}{l}\text { Kluyveromyces } \\
\text { lactis }\end{array}$ & 45 & 3,5 & 5,7 & 23 & 54 \\
\hline \multirow[t]{2}{*}{$\begin{array}{l}\text { Kluyveromyces } \\
\text { marxianus }\end{array}$} & 28 & 2,8 & 6,6 & 18 & 50 \\
\hline & \multicolumn{5}{|c|}{0,8 p. 100 de lactose } \\
\hline $\begin{array}{l}\text { Kluyveromyces } \\
\text { fragilis SG } 1\end{array}$ & 34 & 1,9 & 6,7 & 19 & 55 \\
\hline $\begin{array}{l}\text { Kluyveromyces } \\
\text { fragilis } 5795\end{array}$ & 33 & 1,3 & 9,6 & 15 & 62 \\
\hline $\begin{array}{l}\text { Kluyveromyces } \\
\text { fragilis } 1555\end{array}$ & 35 & 2 & 6,3 & 21 & 68 \\
\hline $\begin{array}{l}\text { Kluyveromyces } \\
\text { fragilis } 397\end{array}$ & 31 & 2,2 & 7,7 & 16 & 57 \\
\hline $\begin{array}{l}\text { Kluyveromyces } \\
\text { lactis }\end{array}$ & 36 & 3,7 & 7,7 & 22 & 58 \\
\hline $\begin{array}{l}\text { Kluyveromyces } \\
\text { marxianus }\end{array}$ & 30 & 3,2 & 8,2 & 13 & 60 \\
\hline
\end{tabular}


lieu de 4,2 p. 100 pour la souche 5795 . On observe donc une plus grande variabilité entre souches pour le métabolisme lipidique que pour le métabolisme protidique.

Après culture en présence de lactose 0,8 p. 100, les différences observées entre souches sont maintenues. La richesse en protéines est en général très légèrement plus faible. On observe par contre une teneur en lipides totaux et en acides gras beaucoup plus élevée après croissance en présence d'un excès de lactose $(0,8$ p. 100) qu'en présence d'une quantité de lactose limitante $(0,2$ p. 100).

Il n'est pas sans intérêt de comparer les résultats des tableaux $\mathrm{n}^{\circ} 1$ et $\mathrm{n}^{\circ} 2$. Lorsque le milieu de culture contient une quantité limitante de lactose $(0,2 \mathrm{p}, 100)$ la composition des cellules est rigoureusement constante et reproductible pour une souche déterminée, quelles que soient nos conditions d'expérience. Il n'en est pas de même en présence d'un excès de lactose $(0,8$ p. 100). Dans ce cas, la multiplication cellulaire s'arrête avant que le lactose et l'azote ammoniacal ne soient entièrement utilisés. Ces cellules en phase stationnaire achèvent de métaboliser le lactose sans se multiplier. Elles peuvent s'enrichir considérablement en protéines (cas des conditions expérimentales du tableau $\mathrm{n}^{\circ} 1$ ), ou non (cas des conditions expérimentales du tableau $n^{\circ} 2$ ) en orientant différemment leurs synthèses. Il sera nécessaire d'étudier systématiquement l'action des facteurs qui diffèrent dans ces deux types d'expérience (volume de la culture - aération).

\section{III. - CONCLUSION}

Les cellules de Kluyveromyces semblent présenter de grandes variations de taille et de composition en fonction des conditions de culture. Malgré ces variations, la comparaison des 6 souches étudiées permet de tirer quelques conclusions.

Kluyveromyces marxianus STV 1043 donne une matière sèche pauvre en protéines et relativement riche en lipides. Le rendement des synthèses protidiques sur lactose est mauvais.

Kluyveromyces lactis SO présente au contraire une grande richesse en protéines et une teneur plus faible en lipides. Malgré ces qualités, cette souche n'a pas d'intérêt industriel car le rendement des synthèses protidiques sur lactose est mauvais.

Les diverses souches de Kluyveromyces fragilis sont plus riches en protéines que $K$. marxianus mais plus pauvre que $K$. lactis. Le rendement exprimé en $\mathrm{mg}$ de protéines formées par $\mathrm{mg}$ de lactose consommé est particulièrement bon pour les souches 5795 et SG 1 . Cette dernière est la plus pauvre en lipides totaux et en acides gras. Cette particularité pourrait faciliter son stockage et sa conservation. 


\section{S u $\mathbf{m} \mathbf{m}$ a r y}

Different types of Kluyveromyces have been compared from two points of view : the proteinic syntheses produce of cells grown on lactose and the composition of the dry matter (proteins and lipids richness).

Reçu pour publication en avril 1972.

Ce travail a fait l'objet d'une aide de la Délégation Générale à la Recherche Scientifique et Technique, contrat $n^{\circ}$ 71-7-3137.

\section{Bibliographie}

Chassang (Aline), Vezinhet (Françoise), Roger (M.) et Galzy (P.). - Variation de la teneur en lipides des cellules de levure au cours de la sporulation. Folia Microbiologica, Prague, sous presse.

STRICKLAND (L. H.) (1951). - The determination of small quantities of bacteria by means of the biuret reaction. J. Gen. Microbiol., 5, 698.

VAN DER WALT (J. P.) (1970). - Kluyveromyces Van der Walt, p. 316 à 378 in The Yeasts a taxonomic study. North Holland Publishing Company, J. Lodder ed. 